\title{
Symmetries in the Kinematic Wave Model and a parameter-free representation of traffic flow
}

\author{
Jorge A. Laval ${ }^{\mathrm{a}, *}$, Bhargava R. Chilukuri ${ }^{\mathrm{a}}$ \\ ${ }^{a}$ School of Civil and Environmental Engineering, Georgia Institute of Technology
}

\begin{abstract}
This paper identifies a family of linear transformations where conservation laws are invariant. In the case of a triangular fundamental diagram, it is shown that for a subset of these transformations, flow, total distance traveled and total delay are invariant. This means that for capacity or delay computations one may choose the transformation -i.e., the shape of the triangular diagram- that simplifies the problem the most, which does not require knowing the actual fundamental diagram. This is appealing also for delay-optimizing control problems since they may be solved using an isosceles fundamental diagram, which provides the most efficient numerical methods. Examples are given.
\end{abstract}

\section{Introduction}

The use of transformations to simplify computations within the kinematic wave model of Lighthill and Whitham (1955) and Richards (1956) has not been a standard tool so far, despite their potential usefulness. The only exception seems to date back to the early 90s, when Gordon Newell (1993) introduced a linear transformation of the time coordinate to simplify the calculations of delays using cumulative count curves. His "moving coordinates" procedure is now the standard method for delay calculations, known as queuing diagrams, where time at each location is measured starting from the passage of an observer moving at free-flow speed.

Recently, a linear transformation of flow and density with remarkable properties was identified independently by Laval and Castrillon (2015) and

\footnotetext{
*Corresponding author. Tel. : +1 (404) 894-2360; Fax : +1 (404) 894-2278

Email address: jorge.laval@ce.gatech.edu (Jorge A. Laval)
} 
Daganzo and Knoop (2015) in the context of the Macroscopic Fundamental Diagram (MFD). Laval and Castrillon (2015) showed that this transformation (i) eliminates all parameters from the kinematic wave model, that (ii) flow is invariant and therefore one may compute capacities in transformed coordinates, and that (iii) the MFD of large networks is symmetric in distribution. In addition, Daganzo and Knoop (2015) used an almost identical transformation to formulate upper and lower bounds for the MFD of pedestrianized streets.

It turns out that these three transformations are strikingly similar, yet they were conceived with very different motivations. They are so useful because by exploiting symmetries of the kinematic wave model. According to Weyl (1952), "An object is symmetrical if one can subject it to a certain operation and it appears exactly the same after the operation. The object is then said to be invariant with respect to the given operation." In the case of the kinematic wave model, we say that it is invariant with respect to the transformations analyzed here because the conservation law looks identical after applying them.

The aim of this paper is to develop a general framework to identify all linear invariant transformations of the kinematic wave model and to analyze the ones that show more promise. We show that the three transformations mentioned above are special cases of a family where flow, total distance traveled and total delay are invariant. This means that one can obtain the solution of a traffic problem using a particular fundamental diagram (FD) that turns the solution simpler to obtain. We say that this turns the kinematic wave model parameter-free because, in the case of triangular FDs, the solution to a wide range of traffic problems can be obtained using any triangular shape for the FD. For example, delay-optimizing control problems may be solved in transformed coordinates without the need to go back to the original coordinates, and therefore no parameters are needed.

The remainder of the paper is organized as follows. Section 2 develops the general framework, which establishes the invariance condition for conservation laws independently of the fundamental diagram. Section 3 analyzes the special case of triangular FDs and shows four transformations that exhibit appealing properties. Section 4 shows three examples that highlight the benefits of these transformations. Finally, section 5 presents a discussion. 


\section{General framework}

In this section we present a general framework that will allow us to identify all symmetry transformations where conservation laws are invariants.

Let the coordinate transformation $(\hat{T}, \hat{X})$ map the original (primal) time and space coordinates $(t, x)$ into transformed (dual) coordinates $(\hat{t}, \hat{x})$, and the state transformation $(\hat{K}, \hat{Q})$ map the primal density, $k(t, x)$, and flow, $q(t, x)$, state variables $(k, q)$ into dual state variables $(\hat{k}, \hat{q})$; i.e.:

$$
(\hat{t}, \hat{x})=(\hat{T}(t, x), \hat{X}(t, x)) \quad \text { and } \quad(\hat{k}, \hat{q})=(\hat{K}(k, q), \hat{Q}(k, q)) .
$$

These transformations are assumed invertible, so that there are maps $(T, X)$ and $(K, Q)$ that satisfy:

$$
(t, x)=(T(\hat{t}, \hat{x}), X(\hat{t}, \hat{x})) \quad \text { and } \quad(k, q)=(K(\hat{k}, \hat{q}), Q(\hat{k}, \hat{q})) .
$$

A conservation law is said to be invariant under transformation group (1) if:

$$
\begin{aligned}
& \hat{k}_{\hat{t}}+\hat{q}_{\hat{x}}=0 \quad \text { when: } \\
& k_{t}+q_{x}=0,
\end{aligned}
$$

where subscripts represent partial derivatives. This means that a dual solution can be mapped into a primal solution using (2).

To obtain the family of transformations that satisfy the symmetry condition (3), we note that $\hat{k}$ can be expressed as a function of $(\hat{t}, \hat{x})$ since $\hat{k}=\hat{K}(k(T(\hat{t}, \hat{x}), X(\hat{t}, \hat{x})), q(T(\hat{t}, \hat{x}), X(\hat{t}, \hat{x}))$; similarly for $\hat{q}$. It can be shown that, by repeated use of the chain rule, the conservation law in transformed coordinates can be expressed as:

$$
\begin{aligned}
\hat{k}_{\hat{t}}+\hat{q}_{\hat{x}}= & \left(T_{\hat{t}} \hat{K}_{k}+T_{\hat{x}} \hat{Q}_{k}\right) k_{t}+\left(X_{\hat{t}} \hat{K}_{k}+X_{\hat{x}} \hat{Q}_{k}\right) k_{x}+ \\
& \left(T_{\hat{t}} \hat{K}_{q}+T_{\hat{x}} \hat{Q}_{q}\right) q_{t}+\left(X_{\hat{t}} \hat{K}_{q}+X_{\hat{x}} \hat{Q}_{q}\right) q_{x} .
\end{aligned}
$$

The key observation here is that if:

$$
\begin{array}{ll}
T_{\hat{t}} \hat{K}_{q}+T_{\hat{x}} \hat{Q}_{q}=0, & X_{\hat{t}} \hat{K}_{k}+X_{\hat{x}} \hat{Q}_{k}=0, \\
T_{\hat{t}} \hat{K}_{k}+T_{\hat{x}} \hat{Q}_{k}=1, & X_{\hat{t}} \hat{K}_{q}+X_{\hat{x}} \hat{Q}_{q}=1,
\end{array}
$$


then (3b) is satisfied. ${ }^{1}$ The system of equations (5) is underspecified and therefore has infinite solutions. Fixing $(\hat{K}, \hat{Q})$ gives a unique solution for $(T, X)$, and vice versa; e.g.:

$$
T_{\hat{t}}=\hat{Q}_{q} / J, \quad T_{\hat{x}}=-\hat{K}_{q} / J, \quad X_{\hat{t}}=-\hat{Q}_{k} / J, \quad X_{\hat{x}}=\hat{K}_{k} / J,
$$

where $J=\hat{K}_{k} \hat{Q}_{q}-\hat{K}_{q} \hat{Q}_{k}$. For the problem to be meaningful we must have:

$$
J \neq 0 \text {. }
$$

Transformation $(T, X)$ can then be obtained by integrating (6). Notice that first-order transformations are the only ones allowed by our formulation. Otherwise, we would have e.g. coordinate transformations that depend on the state variables, e.g. $\hat{T}=\hat{T}(t, x, k, q)$, which are out of the scope of this paper.

\subsection{First-order transformations}

First-order transformations are obtained by setting constant all state transformation derivatives. Let $a_{1}, a_{2}, b_{1}, b_{2}$ be real numbers such that $\hat{K}_{k}=$ $a_{1}, \hat{K}_{q}=a_{2}, \hat{Q}_{k}=b_{1}, \hat{Q}_{q}=b_{2}$. Integration of these quantities and of (6) becomes straightforward and gives:

$$
\begin{array}{lll}
\hat{K}(k, q)=a_{1} k+a_{2} q & \Leftrightarrow & T(\hat{t}, \hat{x})=b_{2}^{\prime} \hat{t}-a_{2}^{\prime} \hat{x}, \\
\hat{Q}(k, q)=b_{1} k+b_{2} q & \Leftrightarrow & X(\hat{t}, \hat{x})=-b_{1}^{\prime} \hat{t}+a_{1}^{\prime} \hat{x},
\end{array}
$$

where $a_{i}^{\prime}=a_{i} / J, b_{i}^{\prime}=b_{i} / J, J=a_{1} b_{2}-a_{2} b_{1}$ and where all constants of integration have been set to zero for simplicity. The remaining transformations can be obtained by inversion; e.g., solving for $(\hat{t}, \hat{x})$ in the system $\{t=T(\hat{t}, \hat{x}), x=X(\hat{t}, \hat{x})\}$ gives $(\hat{T}, \hat{X})$; similarly for $(K, Q)$. Interestingly, this can be expressed as:

$$
\begin{array}{ll}
K(\hat{k}, \hat{q})=T(\hat{k}, \hat{q}), & \hat{T}(t, x)=\hat{K}(t, x), \\
Q(\hat{k}, \hat{q})=X(\hat{k}, \hat{q}), & \hat{X}(t, x)=\hat{Q}(t, x) .
\end{array}
$$

This means that inversion, as defined above, and solving (5) are mathematically equivalent in the case of linear transformations.

\footnotetext{
${ }^{1}$ Condition (5b) could be generalized to $T_{\hat{t}} \hat{K}_{k}+T_{\hat{x}} \hat{Q}_{k}=X_{\hat{t}} \hat{K}_{q}+X_{\hat{x}} \hat{Q}_{q}$, in which case all terms in (6) would have to be multiplied by an arbitrary nonzero constant.
} 
Note that the coefficients $a_{1}, a_{2}, b_{1}, b_{2}$ should not be interpreted as parameters but as arbitrary real numbers. This is true because all these transformations have the invariance property, and therefore can be mapped back to the same primal solution, which is independent of these coefficients. The parameters in our formulation are those introduced by the FD.

\subsection{Measures of performance}

Let $\Psi \equiv \int_{A} k(t, x) d A$ and $\Phi \equiv \int_{A} q(t, x) d A$ be the total time traveled and total distance traveled, respectively, over a time-space region $A$; analogously let $\hat{\Psi} \equiv \int \hat{k}(\hat{t}, \hat{x}) d \hat{A}$ and $\hat{\Phi} \equiv \int \hat{q}(\hat{t}, \hat{x}) d \hat{A}$, with the understanding that $\hat{A}$ is the image of $A$ under the transformation group. We have:

$$
\begin{aligned}
\Psi & =\int_{A} K[\hat{k}(\hat{T}(t, x), \hat{X}(t, x)), \hat{q}(\hat{T}(t, x), \hat{X}(t, x))] d A \\
& =\int K[\hat{k}(\hat{t}, \hat{x}), \hat{q}(\hat{t}, \hat{x})] d \hat{A} /|J| \\
& =K(\hat{\Psi}, \hat{\Phi}) /|J| . \quad \quad \text { (total time traveled) }
\end{aligned}
$$

Notice that the change of variables (1) used in (10b) implies that $d A=d \hat{A} /|J|$, and that the last equality holds due to the linearity of $K$. Similarly,

$$
\Phi=Q(\hat{\Psi}, \hat{\Phi}) /|J| . \quad \text { (total distance traveled) }
$$

This result establishes that the total time traveled and total distance traveled follow the same transformation (8) up to the factor $1 /|J|$.

The total delay in area $A, \Delta$, can be computed as $\Psi-\Psi^{0}$, where the superscript " 0 " indicates free-flow conditions. We have:

$$
\begin{aligned}
& \Delta|J|=\left(b_{2}^{\prime} \hat{\Psi}-a_{2}^{\prime} \hat{\Phi}\right)-\left(b_{2}^{\prime} \hat{\Psi}^{0}-a_{2}^{\prime} \hat{\Phi}^{0}\right) \\
& =b_{2}^{\prime}\left(\hat{\Psi}-\hat{\Psi}^{0}\right)-a_{2}^{\prime}\left(\hat{\Phi}-\hat{\Phi}^{0}\right) \\
& =b_{2}^{\prime} \hat{\Delta}-a_{2}^{\prime}\left(\hat{\Phi}-\hat{\Phi}^{0}\right) \quad \text { (total delay in general) } \\
& =b_{2}^{\prime} \hat{\Delta} \text {. (total delay if system empties) }
\end{aligned}
$$

where the last equality follows since total distance traveled is a constant provided one waits until the system empties, or equivalently, the area $A$ includes all congestion regions in the problem. 
By letting the symbol $A$ also denote its area, Edie's (Edie, 1965) average density, flow and pace in $A, k_{A}, q_{A}$ and $v_{A}$, respectively, are given by:

$$
\begin{aligned}
& k_{A} \equiv \frac{\Psi}{A}=\frac{K(\hat{\Psi}, \hat{\Phi})}{A|J|}=K\left(\hat{k}_{\hat{A}}, \hat{q}_{\hat{A}}\right), \\
& q_{A} \equiv \frac{\Phi}{A}=\frac{Q(\hat{\Psi}, \hat{\Phi})}{A|J|}=Q\left(\hat{k}_{\hat{A}}, \hat{q}_{\hat{A}}\right), \\
& v_{A} \equiv \frac{k_{A}}{q_{A}}=\frac{\Psi}{\Phi}=\frac{K(\hat{\Psi}, \hat{\Phi})}{Q(\hat{\Psi}, \hat{\Phi})},
\end{aligned}
$$

where we used $A|J|=\hat{A}$ and the linearity of $K$ and $Q$. This result is as expected, and it means that average traffic flow variables are subject to the same transformations.

\section{Parameter-free transformations in the kinematic wave model}

The kinematic wave model is the conservation equation (3b) supplemented with the FD:

$$
q=F(k),
$$

which is assumed triangular. It may be defined with a single parameter, as shown in Newell (1993), by measuring flow and density in units of capacity and jam density, respectively. Let the single parameter be $\kappa$, the critical density, and the free-flow pace, $u$, and wave pace, $w$, become $u=\kappa$ and $w=1-\kappa$, respectively. The FD in dual coordinates, $\hat{F}(\hat{k})$, can be obtained by solving the implicit equation $\hat{q}=\hat{Q}(K(\hat{k}, \hat{q}), F(K(\hat{k}, \hat{q})))$ for $\hat{q}$, which gives:

$$
\begin{aligned}
\hat{F}(\hat{k}) & = \begin{cases}\left(b_{1} \kappa+b_{2}\right) \hat{k} /\left(a_{1} \kappa+a_{2}\right) \\
\left.\left((\kappa-1) b_{1}^{\prime}+b_{2}^{\prime}\right) \hat{k}-1\right) /\left(a_{1}^{\prime}(\kappa-1)+a_{2}^{\prime}\right) & \text { if } \hat{k}<\hat{\kappa} \geq \hat{\kappa}\end{cases} \\
\hat{\kappa} & =a_{2}+a_{1} \kappa
\end{aligned}
$$

where $\hat{\kappa}$ is the dual critical density. It is clear that (15) depends on $\kappa$, but this dependency can be eliminated by suitable choices of coefficients $a_{1}, a_{2}, b_{1}, b_{2}$. So far, the only restriction for these coefficients is (7). Now, we add two additional restrictions to ensure that the dual FD be triangular 
and parameter-free: (i) that the dual flow at jam density $\hat{Q}(1,0)=b_{1}$ be zero, in which case the dual capacity becomes $\hat{c} \equiv \hat{Q}(\kappa, 1)=b_{2}$; and (ii) that the dual free-flow pace and wave pace in (15) be constants $\hat{u} \geq 0$ and $\hat{w} \geq 0$ independent of $\kappa$. Restriction (ii) can be accommodated by solving the system $\left\{b_{2} /\left(a_{2}+a_{1} \kappa\right)=1 / \hat{u}, b_{2} /\left(a_{2}+a_{1}(\kappa-1)\right)=1 / \hat{w}\right\}$, obtaining $a_{1}=(\hat{u}+\hat{w}) \hat{c}$ and $a_{2}=(\hat{u}-(\hat{u}+\hat{w}) \kappa) \hat{c}$. Finally, and without loss of generality, we set $\hat{c} \equiv 1$, which implies $\hat{u}=\hat{\kappa}$ and $\hat{w}=(1-\hat{\kappa})$. It follows that the family of all parameter-free transformations can be obtained by setting:

$$
a_{1}=1, \quad a_{2}=\hat{\kappa}-\kappa, \quad b_{1}=0, \quad b_{2}=1 .
$$

or, equivalently:

$$
\begin{aligned}
k & =\hat{k}-(\hat{\kappa}-\kappa) \hat{q}, & q & =\hat{q}, \quad v=\hat{v}-(\hat{\kappa}-\kappa) \\
t & =\hat{t}-(\hat{\kappa}-\kappa) \hat{x}, & x & =\hat{x},
\end{aligned}
$$

where we have used (16), (8), (9) and the definition of pace $v=k / q$. It can be seen that the flow and the position remain invariant, but time and density are not. Time becomes asynchronous, i.e. clocks at each location are set to zero upon the passage of a moving observer traveling at pace $(\hat{\kappa}-\kappa)$, and the density is measured relative to a line of slope $1 /(\hat{\kappa}-\kappa)$, rather than relative to a vertical line as customary. It is worth recalling that $\hat{\kappa}$ should not be interpreted as parameters but as arbitrary positive real numbers, for the same reasons elaborated for $a_{1}, a_{2}, b_{1}, b_{2}$ at the end of section 2.1.

Notice that $J=1$ and thus the area of $A$ and $\hat{A}$ are identical. According to (10c), (11) and (12d) assuming the system empties, the total time traveled, total distance traveled and total delay are given by:

$$
\Psi=\hat{\Psi}-(\hat{\kappa}-\kappa) \hat{\Phi}, \quad \Phi=\hat{\Phi}, \quad \Delta=\hat{\Delta}
$$

It follows from (17a) and (18) that flows, total distance traveled and total delay are all invariant. The equation for the total travel time in (18) can be divided by $\Phi$, combined with (13c) and rearranged to obtain:

$$
v_{A}-\kappa=\hat{v}_{\hat{A}}-\hat{\kappa},
$$

which is identical to the pace in (17a). This means that the average travel time (per unit distance) in excess of the free-flow travel time is also invariant. Again, if the system empties this quantity would correspond to the average delay.

As shown next, all transformations existing in the traffic flow literature are obtained by varying $\hat{\kappa}$. 


\subsection{Newell's transformation}

Newell's transformation (Newell, 1993, p. 292) is obtained by setting $\hat{\kappa}=0$, and produces the dual FD shown in Fig. 1a, which exhibits an infinite free-flow speed. This means that in free-flow conditions vehicles travel infinitely fast and that in congestion their travel time equals their delay. The geometrical interpretation of this transformation has become the standard method for calculating delays when arrival and departure curves are known; i.e. shift the arrival curve to the right by the free-flow trip time between the two locations and the area between the two becomes the total delay.

The mirror image of Newell's transformation, which has not appeared in the literature to the best of our knowledge, is obtained by setting $\hat{\kappa}=1$; see Fig. 1b. Here the wave speed is infinite, which means that under congestion waves travel infinitely fast upstream. The geometrical interpretation here is that when the departure curve is under congestion it can be shifted vertically upwards by the jam accumulation between the two locations.

\subsection{Isosceles transformation}

From a practical perspective, isosceles FDs are very convenient, especially when it comes to numerical solution methods. Within our framework, it simply means that $\hat{\kappa}=1 / 2$, and this corresponds to the transformation in Daganzo and Knoop (2015).

\subsection{Isosceles transformation with offset}

The transformation in Laval and Castrillon (2015) adds an offset to the density, i.e.:

$$
k=\hat{k}-(\hat{\kappa}-\kappa) \hat{q}-1 / 2
$$

which is possible since constant terms correspond to integration constants in our framework, which do not alter the invariance property of the transformations, as noted below eqn. (9). The advantage of this transformation is that it is the only one where the passing rate of forward and backward wave speed are identical and equal to 1. As shown in Laval and Castrillon (2015) this simplifies variational theory significantly.

In this case $\hat{v}=\hat{q} /(\hat{k}+1 / 2)$ and therefore $v=\hat{v}-(\hat{\kappa}-\kappa)$ still applies. The only difference is that $-a_{3} b_{2} \hat{A}=\hat{A} / 2$ should be added to the total travel time, but total distance traveled and total delay remain unchanged:

$$
\Psi_{A}=\hat{\Psi}-(\hat{\kappa}-\kappa) \hat{\Phi}+\hat{A} / 2, \quad \Phi=\hat{\Phi}, \quad \Delta=\hat{\Delta} .
$$




\section{Examples}

In this section we show three examples that illustrate the main concepts introduced in this paper. In all cases we solve a traffic problem both in primal and dual coordinates to show that the measures of performance are related according to the theory proposed here.

\subsection{A single bottleneck}

Consider an uncongested freeway with a constant flow $\lambda$ when a bottleneck of capacity $\mu<1$ appears for a duration $\tau$. Fig. 1 presents the standard kinematic waves solution of the problem in the flow-density plane (first column) and the time-space plane (second column) using Newell's transformation (first row), Newell's mirror image transformation (second row) and the isosceles transformation (third row). The solution with a general triangular FD has been overlaid to each row using dashed lines. It can be seen that the maximum queue length (2.5 length units) and the duration of congestion at each location (3.5 time units at $x=0$ ) are identical in all figures, which explains why delays are invariant. Formally, based on this figure it can be shown that:

$$
\begin{array}{ll}
\Psi=A / 2-(1 / 2-\kappa) A \lambda, \quad & \Phi=A \lambda \\
\Delta=(1-\lambda) A / 2, \quad \text { where } & A=\frac{(1-\mu)(\lambda-\mu) \tau^{2}}{(1-\lambda)^{2}}
\end{array}
$$

which applies to both primal and dual coordinates; for dual coordinates one has to "put a hat" on all relevant variables. Notice that area $A$ (as defined in the last row the figure), the total distance traveled and the total delay are independent of the FD; i.e. they are invariant, as expected. For the total travel time, next we show that (18) is satisfied for all transformations.

Under Newell's transformation these measures of performance may be obtained simply by setting $\kappa=0$ in (22), since this defines the FD associated with Newell's transformation. This implies $\hat{\Psi}=A / 2+\lambda A / 2$. Using this result in (18) we get $\Psi=A / 2+\lambda A / 2-(1-\kappa) A \lambda=A / 2-(1 / 2-\kappa) A \lambda$, which corresponds to (22a), as sought.

For the other transformations the procedure is similar. Under Newell's mirror image and Daganzo and Knoop (2015)'s isosceles transformations we set $\kappa=1$ and $\kappa=1 / 2$ in (22), respectively, and the reader can verify that (18) is satisfied too. For Laval and Castrillon (2015)'s transformation one would have to solve the problem from scratch (because (22) does not account for the density offset) to obtain $\hat{\Psi}=0$, which also satisfies $(21)$. 


\subsection{Random bottlenecks}

This example shows that the proposed theory works also for stochastic problems, by showing that the distribution of delays are the same in primal and dual coordinates. The setting is similar to the above but now there is a stream of bottlenecks that appear randomly in space and time according to a Poisson process of rate $f$, in units bottlenecks/time-space. The corridor is an open system with flow entering the system at its upstream boundary and exiting the system at its downstream boundary. This scenario could be appropriate for modeling weaving sections or pedestrian crossings as in Daganzo and Knoop (2015), who also provides bounds for the resulting MFD. Here, we tackle the solution with simulation to highlight the practical usefulness of the transformations.

The traffic simulation used here gives the exact numerical solution of the kinematic wave model with a triangular FD. It can be categorized as a mesoscopic car-following model where each trajectory represents a group of vehicles, and is described in Laval and Leclercq (2013).

All the primal experiments in this section correspond to an initial and boundary value problem (IBVP). The boundary value is the flow $\lambda$ entering the road segment and the initial density is $k_{0}=\lambda \kappa$. We use $\kappa=1 / 7$ for the primal FD, and $\hat{\kappa}=1 / 2$ for the dual (isosceles) FD. Notice that homogeneous Poisson processes remain invariant since our transformations conserve areas. Next, we analyze two scenarios that highlight different aspects of the transformation.

Scenario 1 helps understand better why these transformations work; see Fig. 2: we (i) start with a given set of initial and boundary conditions, and bottleneck realizations for the primal problem (left), (ii) perform their transformation to obtain boundary and bottleneck data for the dual problem (right), and finally (iii) run the simulation in both cases to obtain the simulated trajectories shown in the figures. Notice that the dual problem is no longer an IBVP since the initial data in the primal changed to data along the moving observer line in the dual. This can be seen by the bold circles in the figure, which represent initial vehicle positions in the primal problem, and which map to the corresponding circles in the right column. The difference between the top and bottom rows of the figure is in the number of bottlenecks and in the number of vehicles that a trajectory represents in the dual problem (1 and 2 , respectively). The boundary flow drops to zero after a prescribed time and the simulation ends when the last entering vehicle exits 
the segment. The areas $A$ and $\hat{A}$ correspond to the unshaded areas in the figures.

Comparing the left and right columns it can be seen that the distance traveled by each vehicle, the delay of each vehicle, and bottleneck queue lengths are all identical, as expected. That delays are the same can also be explained by noting that at a fixed location, time intervals are invariant in our transformations. The time traveled by each vehicle are not, also as expected from the theory; from the figure it can be seen that the reason is in the difference in the time spent at free-flow speed ( $\hat{\kappa}-\kappa$ per unit distance), since we already noted that at a fixed location time intervals, i.e. delays in this case, are invariant.

Scenario 2 relaxes the exact correspondence between primal and dual simulation settings; see Fig. 3. This scenario highlights the practical usefulness of the proposed transformations by simulating an IBVP for both the primal and the dual problems, and showing that outputs are still as predicted by the theory. In this scenario, the dual initial conditions are the transformed primal ones, as before, but now we stop the simulation at a prescribed time in both cases. This means that $A=\hat{A}$, and they correspond to the entire time-space region being simulated; i.e. they are not the transformation of each other. Similarly, dual bottlenecks are a realization independent from the primal bottlenecks. As can be seen in the figure the primal and dual simulation output seem very different. But the measures of performances are as predicted by the theory. To see this, Fig. 4 presents box-whiskers plots for the total delay $\Delta$ across 30 repetitions of this experiment, and for different values of $f$ and $\lambda$. Since the system is not emptied, we show the primal total delay using two methods: (i) directly from the simulation, in orange and (ii) using (12c), in blue. It can be seen that all intervals overlap, including the confidence intervals for the mean shown as gray rhombi, and therefore we can say that both the primal and dual problems yield statistically equivalent results.

\subsection{Optimal ramp-metering control}

This final example illustrates that traffic control problem that minimize total delays can be solve in transformed coordinates altogether, which does not require FD parameters.

The traffic flow problem considered here is identical to the one in Laval and Leclercq (2010), but now on-ramps are metered and optimized for minimal delay using an optimal control formulation that closely follows Gomes 
and Horowitz (2006). In a nutshell, we consider a long freeway corridor with continuum inflow and outflow demand rates in time and space. On-ramps are finite in length and traffic dynamics given by the kinematic wave model. The optimal control problem was run for three different FDs, i.e. $\kappa=1 / 7$ and $1 / 2$. Fig. 5(left) shows the optimal ramp-metering rates in space and time for $\kappa=1 / 7$; the surfaces for $\kappa=1 / 2$ is virtually identical, as expected. To see this, Fig. 5(right) shows a cross-section of the surface for fixed times, where the overlap is apparent.

\section{Discussion}

We have identified a family of symmetry transformations of the kinematic wave model with arbitrary FD. In the case of triangular FDs, flows and total delay are invariant, which means that all triangular shapes produce the same solution in terms of flows and delays provided that both time and initial data, if any, are transformed using (17). These findings can be advantageous in at least the following areas:

(i) Analytical computations. For capacity or delay computations one may choose the FD that simplifies the problem the most to carry out the calculations, which does not require knowing the facility's FD. The best example of this approach is, as mentioned earlier, Newell's method to compute delays. Another example is looking at the dynamics of "holes" -or absence of vehicles- in the freeway rather than the vehicles themselves, an argument origin used by Newell (1993) to simplify the solution of inhomogeneous freeway problems. Since holes travel upstream at pace $w$ and produce waves that travel downstream at pace $u$, their FD is a mirror image of the diagram for regular vehicles. Within our framework this means $\hat{\kappa}=1-\kappa$ or $a_{2}=1-2 \kappa$, which formally proves why the holes approach works. It is expected that the generalized framework proposed here will open the door for efficient solution methods for other traffic flow problems.

(ii) Numerical computations. The efficiency of existing numerical tools based on triangular FDs might be increased dramatically by using an isosceles FD. For example, under an isosceles diagram (a) Godunov's method (e.g., CTM model) becomes exact (Leclercq et al., 2007), (b) methods based on variational theory no longer require memory since forward and backward wave speeds are identical, and (c) methods based on cellular automata (CA) may be implemented using CA rule 184, which is extremely computationally efficient. 
(iii) Optimal control problems seeking minimizing delay or total distance traveled or vehicle accumulation in the context of the MFD, should benefit from (ii) above not only from faster execution, but possibly from an improved formulation devoid of memory.

The proposed theory may be considered parameter-free for boundaryvalue problems where an invariant quantity is sought (delay, flow or total distance traveled); no parameters are needed since the initial data nor the final answer have to be transformed. This can be very useful for delayoptimizing control problems and for estimating system capacity. Of course, if we require field implementation one needs to transform the dimensionless answer to a dimensional one, e.g. transform the dimensionless metering rates to veh/hr, which requires knowing the capacity. The important point here is that, for a given set of initial conditions, the ramp-metering strategy that minimizes total delay, as a proportion of capacity, is independent of capacity. Problems containing bottlenecks (e.g. traffic lights) require knowing $\kappa$ if their trajectories are to be transformed; but as shown in $\S 4.2$ if only their distributions are known and stationary, then no parameters are needed.

There are other transformations that might be useful, albeit not parameterfree and where the dual FD is not triangular. One example are densityconserving transformations such as:

$$
\begin{aligned}
k & =\hat{k}, & q & =v_{0} \hat{k}+\hat{q}, \\
t & =\hat{t}, & x & =v_{0} \hat{t}+\hat{x},
\end{aligned}
$$

where $v_{0}$ is a parameter. Following $\S 2.2$ the reader can verify that in this case both the total delay and total time traveled are invariant, but not the total distance traveled. Nonlinear transformations can also be useful, although it was noted that they would imply the more complicated form $\hat{T}=\hat{T}(t, x, k, q)$. These and other transformations are currently being investigated by the authors.

\section{Acknowledgments.}

This research was supported by NSF research project 1301057 and Georgia DOT project $2006 \mathrm{~W} 20$. The authors would like to thank two anonymous reviewers whose comments greatly improved the quality of this paper. 


\section{References}

Daganzo, C., Knoop, V. L., 2015. Traffic flow on pedestrianized streets. Transportation Research Part B: Methodological (Forthcoming).

Edie, L. C., 1965. Discussion of traffic stream measurements and definitions. In: 2nd Int. Symp. on Transportation and Traffic Theory. Paris, France, pp. $139-154$.

Gomes, G., Horowitz, R., 2006. Optimal freeway ramp metering using the asymmetric cell transmission model. Transportation Research Part C: Emerging Technologies 14 (4), $244-262$.

Laval, J., Castrillon, F., 2015. Stochastic approximations for the macroscopic fundamental diagram of urban networks. Transportation Research Part B: Methodological 81 (3), 904-916.

Laval, J. A., Leclercq, L., 2010. Continuum approximation for congestion dynamics along freeway corridors. Transportation Science (44), 87-97.

Laval, J. A., Leclercq, L., 2013. The Hamilton-Jacobi partial differential equation and the three representations of traffic flow. Transportation Research Part B: Methodological 52, 17-30.

Leclercq, L., Laval, J., Chevallier, E., 2007. The Lagrangian coordinate system and what it means for first order traffic flow models. In: Heydecker, B., Bell, M., Allsop, R. (Eds.), 17th International Symposium on Transportation and Traffic Theory. Elsevier, New York.

Lighthill, M. J., Whitham, G., 1955. On kinematic waves. I Flow movement in long rivers. II A theory of traffic flow on long crowded roads. Proceedings of the Royal Society of London 229 (A), 281-345.

Newell, G. F., 1993. A simplified theory of kinematic waves in highway traffic, I general theory, II queuing at freeway bottlenecks, III multi-destination flows. Transportation Research Part B 27 (4), 281-313.

Richards, P. I., 1956. Shockwaves on the highway. Operations Research (4), $42-51$.

Weyl, H., 1952. Symmetry. Princeton University Press. 


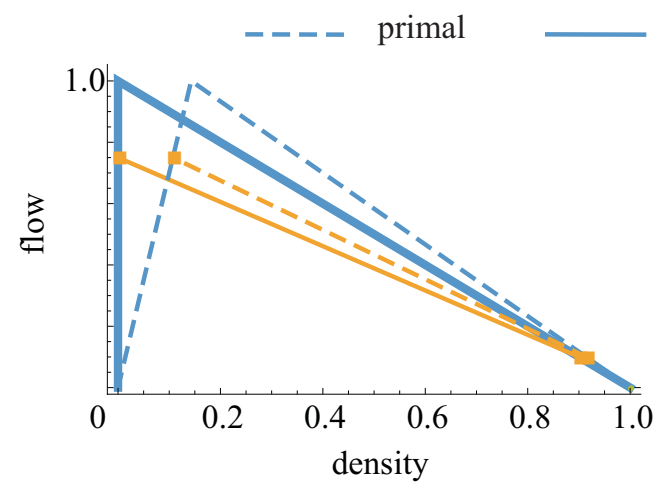

dual
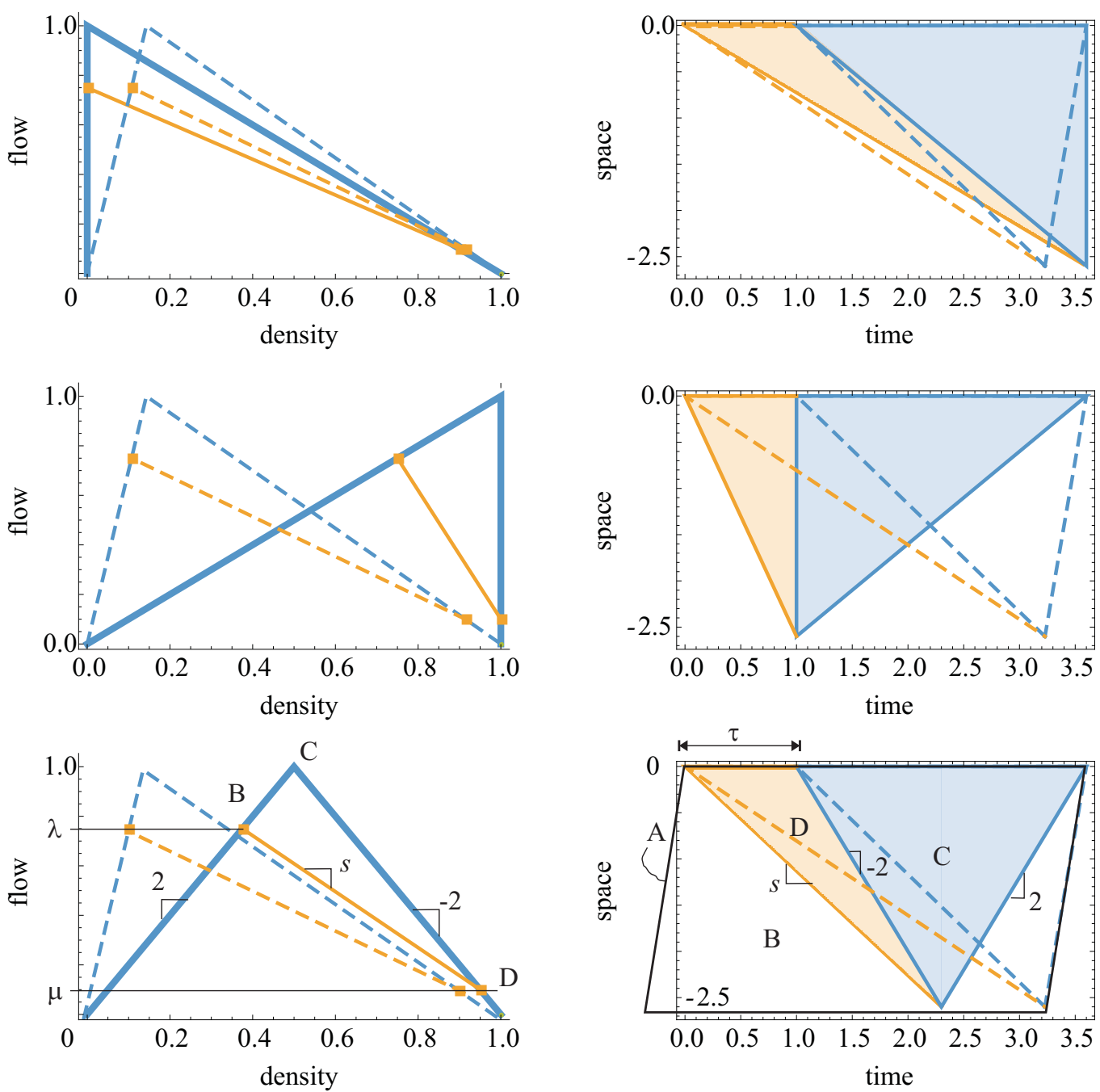

Figure 1: The single bottleneck example. 

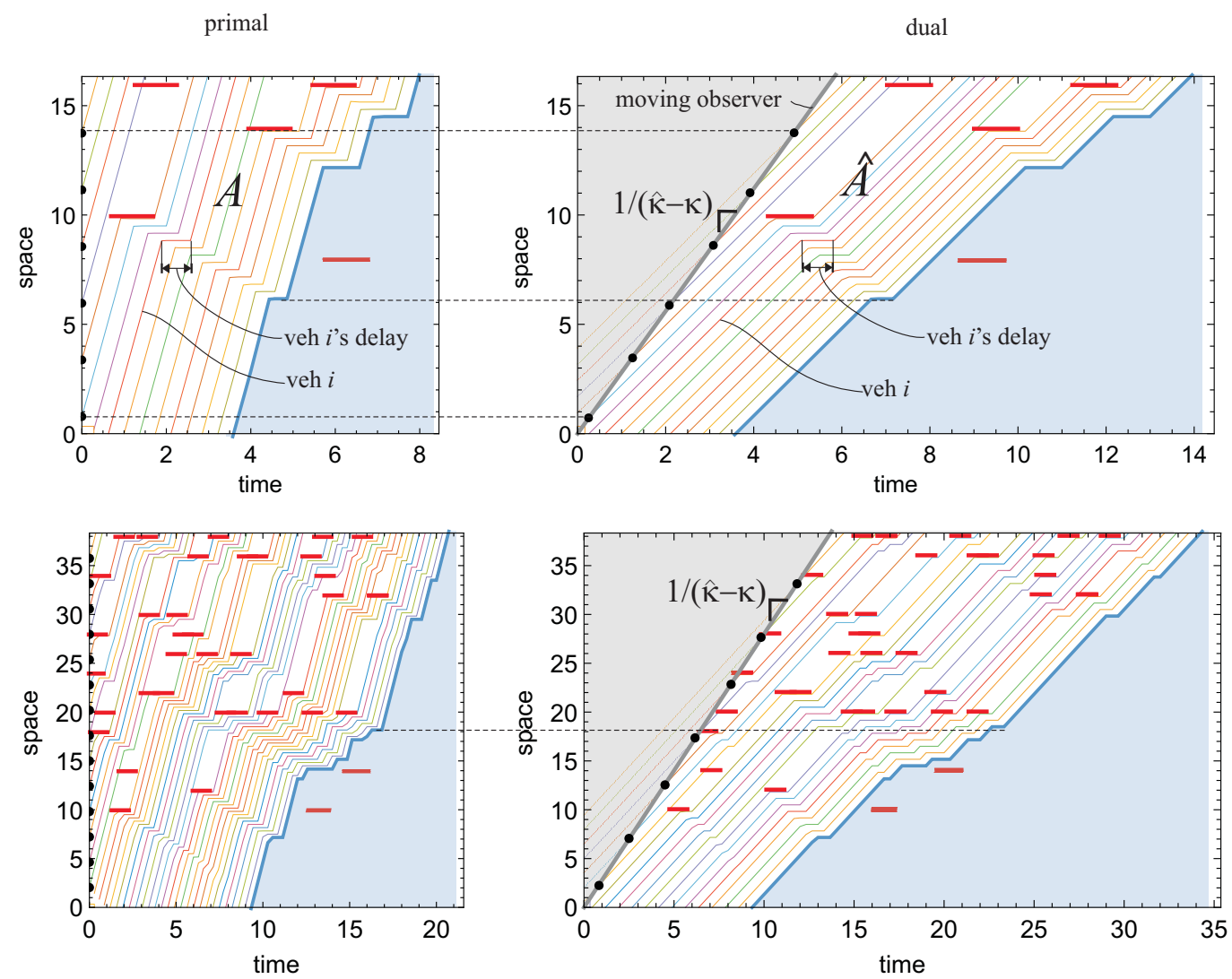

Figure 2: The random bottlenecks example: sample simulation output for scenario 1. 

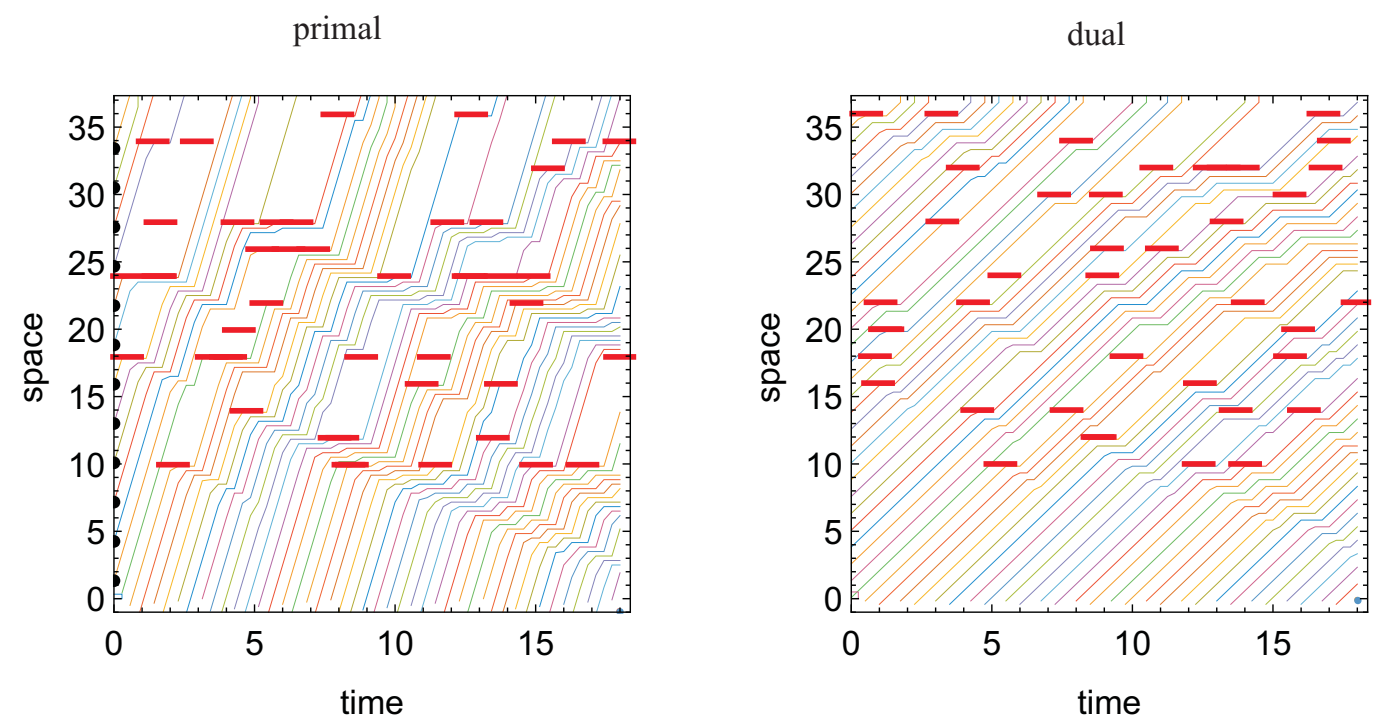

Figure 3: The random bottlenecks example: sample simulation output for scenario 2 . 

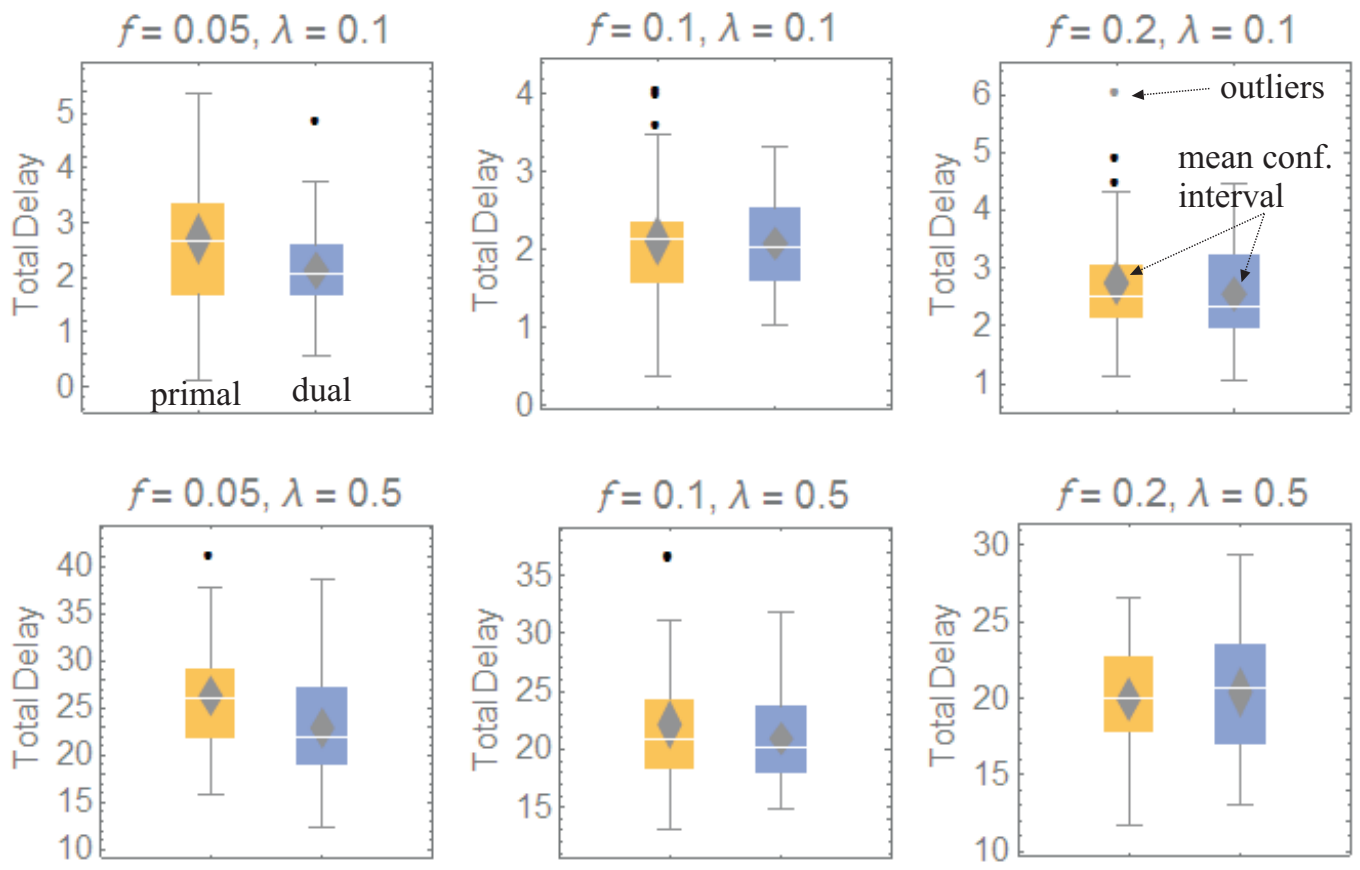

Figure 4: The random bottlenecks example, scenario 2: box-whiskers plots for the total delay $\Delta$ across 30 repetitions of this experiment, and for different values of $f$ and $\lambda$. 

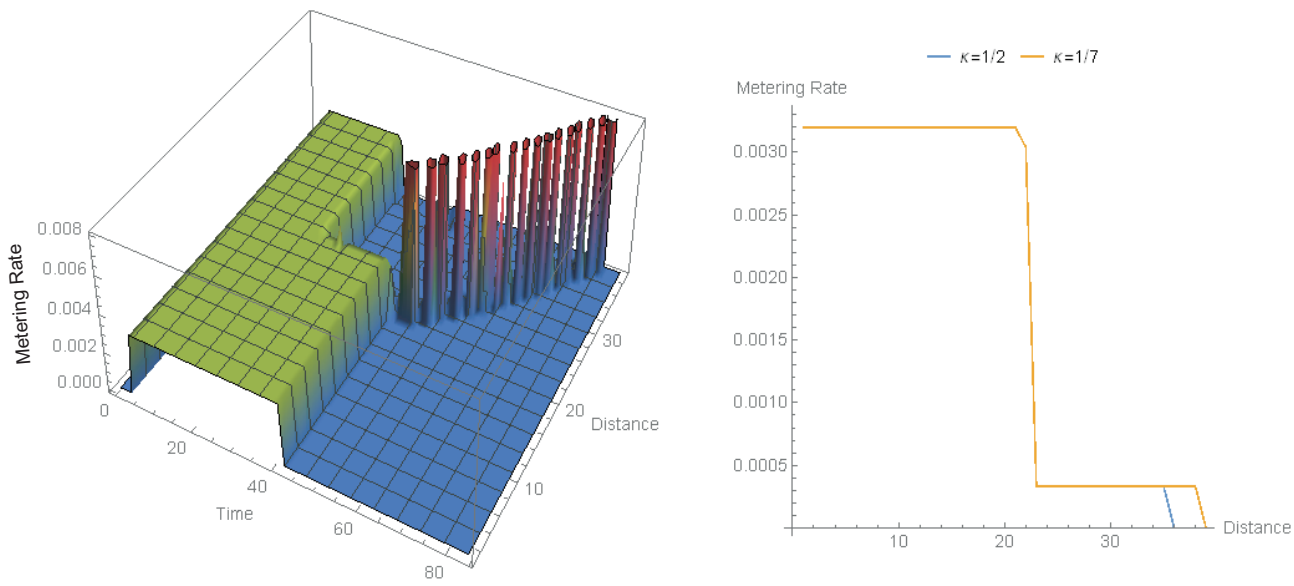

Figure 5: Optimal ramp-metering control example: (left) the optimal ramp-metering rates in space and time for $\kappa=1 / 7$; (right) cross-section of the surface for fixed times. The on-ramp demand is $50 \%$ of one lane capacity, the exit probability is $0.1 / 150$ over a distance of one jam spacing, and the spatial discretization is 15 jam spacing units. On ramps were loaded during the first 40 time steps and the optimization ends when the system is empty. 